\title{
A viabilidade da metodologia Pragmatic Randomized Clinical Trials (PRCT) à pesquisa de Medicina Tradicional Chinesa
}

\author{
The feasibility of the Pragmatic Randomized Clinical Trials (PRCT) for researching Tradicional \\ Chinese Medicine
}

\author{
Bruna Verzili Gallo ${ }^{1^{*}} \bullet$, Ana Claudia de Leite-Mor ${ }^{2} \bullet$ \\ ${ }^{1}$ Graduanda do curso de Naturologia da Universidade Anhembi Morumbi, São Paulo, São Paulo, Brasil. ${ }^{2}$ Docente do curso de graduação \\ em Naturologia na Universidade Anhembi Morumbi, São Paulo, São Paulo, Brasil. *Autor para correspondência. E-mail: \\ brunaverzili@gmail.com
}

\begin{abstract}
Resumo: Introdução: A Medicina Tradicional Chinesa (MTC) é uma Prática Integrativa e Complementar e emprega uma visão integrativa e complexa sobre o sujeito. A metodologia dos Randomized Controlled Trials não contemplam a visão complexa da MTC. No entanto, outros métodos como os Pragmatic Randomized Controlled Trials parecem ser mais adequados. O objetivo do trabalho foi sistematizar e discutir os artigos que utilizaram a metodologia Pragmatic Randomized Controlled Trials com práticas da MTC. Revisão: Foi realizada uma revisão sistemática da literatura de ensaios clínicos que utilizaram a metodologia de PRCT. Foram levantados artigos das bases de dados: PubMed, Scielo, Mosaico, Web Of Science e Cochrane. Discussão: Os 17 estudos selecionados verificaram eficácia da MTC, em comparação com o tratamento usual biomédico. A PRCT parece ser uma metodologia muito adequada para a realização de ensaios clínicos e produção de evidências quanto às práticas da MTC e demais práticas integrativas e complementares. Considerações finais: a metodologia PRCT permite a implicação de uma visão multidimensional e singular do sujeito, oferecendo o benefício de não excluir a complexidade da terapêutica e a visão singular do profissional da saúde em relação aos pacientes tratados na pesquisa.
\end{abstract}

Palavras-chave: terapias complementares, medicina integrativa, medicina tradicional chinesa, ensaio clínico pragmático.

\begin{abstract}
Introduction: Traditional Chinese Medicine (TCM) is an Integrative and Complementary Practice and employs an integrative and complex view of the subject. Randomized Controlled Trials' methodology does not address the complex view of TCM. However, other methods such as pragmatic randomized controlled trials seem to be more appropriate. Objective of this work was to systematize and discuss the articles that used the Pragmatic Randomized Controlled Trials methodology with TCM practices. Review: A systematic review of the literature of clinical trials using the PRCT methodology was performed. Articles from the databases were collected: PubMed, Scielo, Mosaico, Web Of Science and Cochrane. Discussion: The 17 selected studies found efficacy of TCM compared to the usual biomedical treatment. The PRCT seems to be a very appropriate methodology for conducting clinical trials and producing evidence regarding TCM practices and other integrative and complementary practices. Final considerations: the PRCT method allows the implication of a multidimensional and singular view of the subject, offering the benefit of not excluding the complexity of the therapy and the singular view of the health professional in relation to the patients treated in the research.
\end{abstract}

Keywords: complementary therapies, integrative medicine, pragmatic clinical trial, medicine, chinese traditional, methods, research design.

\section{Introdução}

A Medicina Tradicional Chinesa (MTC) foi incluída na Política Nacional de Práticas Integrativas e Complementares (PNPIC) do Brasil no ano de 2006, para complementar os tratamentos da atenção primária em saúde. Levando isso em conta, necessitam-se de pesquisas e ensaios clínicos que estabeleçam para a MTC e para as demais Práticas Integrativas e Complementares (PIC) padrões de eficácia, segurança e efetividade, em vista de aprimorar a aplicação dessas terapias no sistema público de saúde brasileiro (BRASIL, 2006). O presente trabalho objetivou analisar a metodologia de ensaio clínico Pragmatic Randomized Controlled Trials (PRCTs) em vista de debater sua viabilidade para a produção de evidências da MTC e das demais PIC. 
As PIC são tratamentos que utilizam recursos terapêuticos baseados em conhecimentos tradicionais caracterizados por uma visão integral do sujeito (mente, corpo, espírito, aspecto vital). Apresentam uma abordagem complexa, não fragmentada e reducionista, em relação ao caso clínico e apresentam uma considerável qualificação da relação entre terapeuta-paciente. São práticas que priorizam a prevenção de doenças e a promoção de saúde, bem como o aumento da qualidade de vida, a redução do estresse, da ansiedade e dos sintomas em caso de doença crônica (Brasil, 2006; Resch, 1998; Contatore et al., 2015; Lu et al., 2004).

A Política Nacional de Práticas Integrativas e Complementares (Brasil, 2006) vem sendo aprimorada a cada ano, para garantir a integralidade na atenção à saúde no SUS, e a sua implementação envolve justificativas de natureza política, técnica, econômica, social e cultural. Esta política atende, sobretudo, à necessidade de se conhecer, apoiar, incorporar e implementar experiências que já vêm sendo desenvolvidas na rede pública de muitos municípios e estados, entre as quais destacam-se aquelas no âmbito da Medicina Tradicional Chinesa: a acupuntura, a auriculoterapia e as práticas corporais (BRASIL, 2006; Zhufan, 2008; Maciocia \& Ming, 1996).

A metodologia reconhecida como padrão ouro na produção de evidências são os chamados Randomized Controlled Trials (RCT). Esse método de ensaio clínico exige que sejam padronizados protocolos clínicos e, para o cegamento, que o pesquisador não saiba em quais participantes está aplicando a terapia ou placebo. Estas exigências não podem ser eficientemente aplicadas às pesquisas com Práticas Integrativas e Complementares (PIC) (Souza \& Luz, 2011; Rocha \& Gallian, 2013), visto que estas práticas pressupõem um tratamento individualizado e não padronizado, ao mesmo tempo que, muitas delas, não são passíveis de cegamento.

A abordagem terapêutica da MTC consiste em uma visão complexa do sujeito que leva em conta a singularidade de seu processo de saúde-adoecimento nas dimensões mental, emocional, metabólica, física e vital (Zhufan, 2008; Maciocia \& Ming, 1996), impossibilitando a padronização das intervenções nessa terapêutica (Rocha \& Gallian, 2013; Tabosa, 2012). Nesse sentido, é necessário que se desenvolvam novas metodologias de pesquisa mais adequadas aos princípios e características das PIC (Andrade \& Portella, 2018). A metodologia de pesquisa Pragmatic Randomized Controlled Trials (PRCTs) é uma opção que pode suprir essa demanda, pois permitem a singularização dos protocolos terapêuticos e a diversificação de práticas utilizadas, respeitando a complexidade das PIC (Coutinho \& Bloch, 2003; Roland \& Torgerson, 1998; Resch, 1998). Os ensaios pragmáticos são úteis para responder a perguntas sobre a eficácia de uma terapia quando comparados a algum tratamento padrão ou aceito, não com um placebo. Eles também superam algumas dificuldades específicas que podem ser encontradas com ensaios explicativos de terapias complementares (PIC), por exemplo, ao avaliar pacotes completos de cuidados (MacPherson, 2004).

Apesar de se considerar que os estudos pragmáticos não fornecem informações conclusivas sobre a especificidade do efeito de determinados tratamento, podemos observar algumas características interessantes: (a) o desenho de um estudo pragmático reflete variações entre pacientes que ocorrem na prática clínica real e não em circunstâncias clínicas reais artificiais, aproximando a pesquisa científica da complexidade do contexto clínico (Roland \& Torgerson, 1998; Ware \& Hamel, 2011; Huf \& Kritski, 2012); (b) são mais aderentes à prática convencional do tratamento sob investigação (Ware \& Hamel, 2011; Moreira et al., 2013); (c) geralmente são mais simples e mais baratos do que ensaios explicativos (Moreira et al., 2013; Huf \& Kritski, 2012); (d) respondem diretamente à pergunta (fundamental para o profissional da saúde): "devo propor este tratamento para um paciente pela condição X (Roland \& Torgerson, 1998; MacPherson, 2004; Huf \& Kritski, 2012)”; (e) avaliam a efetividade de um tratamento, ao invés de sua eficácia (Coutinho \& Bloch, 2003; Huf \& Kritski, 2012); (f) podem incorporar uma população heterogênea de pacientes; (g) os regimes de tratamento tendem a ser mais flexíveis, acomodando assim as necessidades individuais dos pacientes (Coutinho \& Bloch, 2003).

Segundo as diretrizes descritas na PNPIC, existe a necessidade de aprimoramento do conhecimento científico sobre as práticas da MTC, bem como sobre outras PIC. Entretanto, consideramos ser inadequado supor que critérios de avaliação da eficácia da racionalidade biomédica, tal como os empregados nos RCT, sejam aplicáveis a outras racionalidades, como a MTC (Andrade \& Portella, 2018). A pesquisa em MTC é um desafio metodológico que tem sido realizado, majoritariamente, através em ensaios clínicos estatísticos (Souza \& Luz, 2011). Entretanto, pode-se encontrar diversos ensaios clínicos para mostrar a efetividade das terapias da Medicina Tradicional Chinesa (MTC) que se utilizam da metodologia PRCT, sendo que o mesmo não ocorre para outras PIC.

O presente estudo trata de uma revisão sistemática integrativa da literatura que selecionou ensaios clínicos que testaram práticas da MTC, através da metodologia de PRCT. Nosso objetivo foi refletir sobre a viabilidade desta metodologia e sua adequação às características da racionalidade da MTC e das PIC em geral. 


\section{Revisão}

Foi desenvolvida uma revisão sistemática integrativa da literatura (Ercole et al., 2014) que buscou selecionar na literatura internacional estudos de PRCT que tenham sido aplicados a práticas da MTC. O levantamento bibliográfico foi realizado no mês de maio de 2019, onde foram consultadas as seguintes bases de dados: PubMed, Scielo, Cochrane, Web of Science e Mosaico. Dezessete palavras-chave, da língua inglesa, foram utilizadas nas barras de busca, considerando as aspas: "pragmatic trial", "pragmatic trial design", "pragmatic trial" (AND) "complementary and alternative medicine", "pragmatic trial" (AND) CAM, "pragmatic trial” (AND) "traditional medicine", "pragmatic trial” (AND) TM, "pragmatic trial” (AND) "integrative medicine", "pragmatic trial" (AND) "integrative health", "pragmatic trial" (AND) "naturopathy", "pragmatic research design", "pragmatic research", "pragmatic research" (AND) "complementary and alternative medicine", "pragmatic research" (AND) CAM, "pragmatic research" (AND) "traditional medicine", "pragmatic research" (AND) TM.

Ao todo, 1.788 artigos foram levantados. Desses, foram selecionados, através de leitura do título e resumo, os que empregaram a metodologia de PRCT e que tenham testado uma ou mais PIC, totalizando após a eliminação das duplicatas, 162 artigos. Desses, 45 eram revisões sistemáticas da literatura e foram excluídas da análise. Restaram 117 artigos que se tratavam de ensaios clínicos. Desses, foram selecionados aqueles que estudaram práticas da MTC, totalizando 21 artigos lidos na íntegra, por ser a racionalidade em predominância de artigos. Destes 21, quatro estudos não haviam de fato desenvolvido um PRCT, de forma que apenas 17 foram selecionados para leitura revisional rigorosa, que avaliou a pertinência desta metodologia em acordo aos princípios e características da racionalidade terapêutica da MTC (Figura 1).

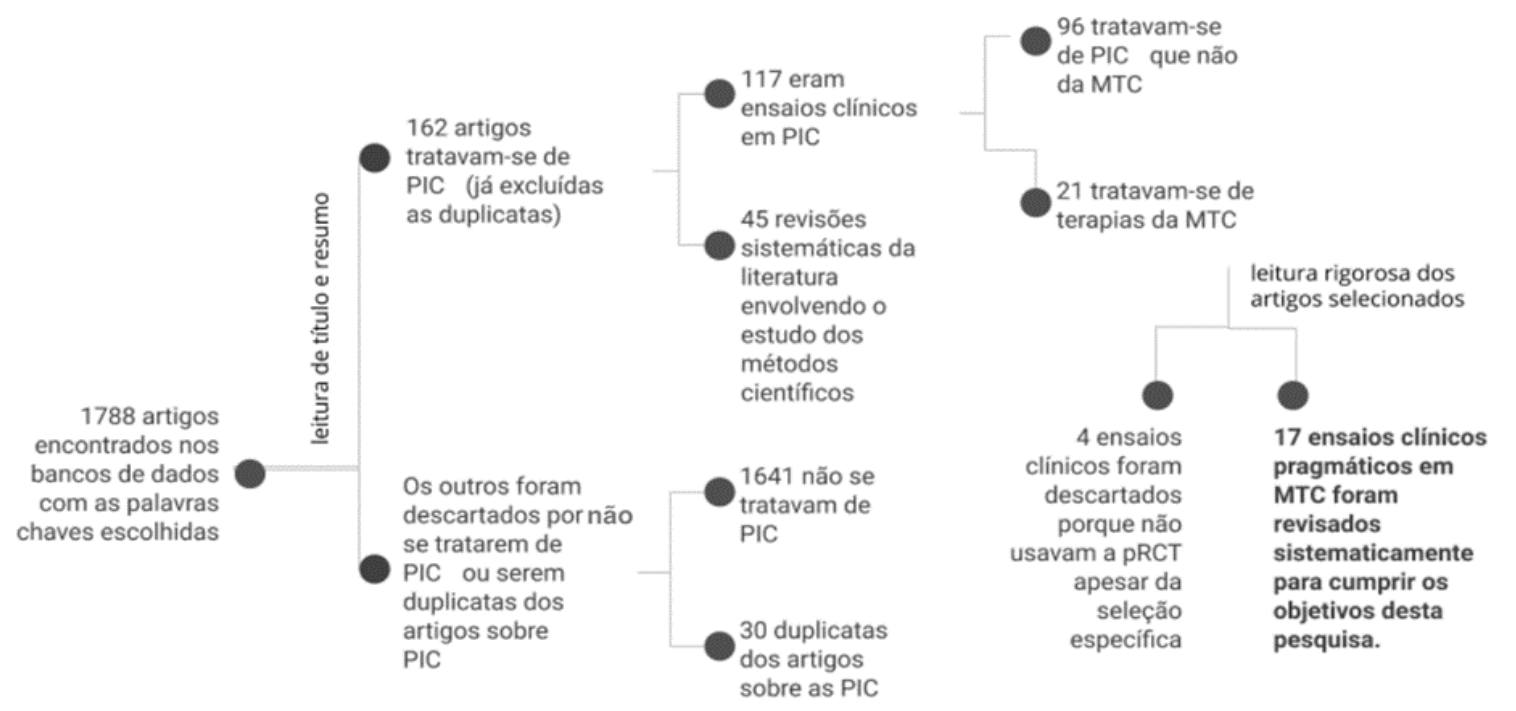

Figura 1. Fluxograma do levantamento bibliográfico e seleção dos artigos.

\section{Discussão}

Como explicado em metodologia, não se utilizou nesta revisão um critério de inclusão ou exclusão por ano de publicação, a fim de maximizar o número de artigos a serem revisados. Verifica-se que, dos 17 artigos selecionados, suas publicações ocorreram entre 2004 e 2019, sendo que 13 (76\%) são dos últimos 10 anos e 8 dos últimos 5 anos - o que indica o crescente interesse na PRCT para mostrar a eficiência da MTC no cenário clínico. Diversas técnicas terapêuticas oferecidas pela MTC foram utilizadas nos artigos revisados: acupuntura (12), massoterapia e quiropraxia (1), moxabustão (2) e fitoterapia (2).

Ao analisar as especificidades metodológicas dos artigos, constatou-se que quinze artigos (88\%) não utilizaram placebo como grupo controle - de acordo com o método PRCT. Desses 15, um deles explica que, sem um grupo placebo, com simulação de intervenções com acupuntura (sham acupuncture), essa análise não pode diferenciar aspectos do tratamento com acupuntura entre resultados específicos e inespecíficos (Reynolds et al., 2008).

Do total, oito artigos (47\%) utilizaram, no grupo de teste, apenas a intervenção com medicina chinesa e quatro (23,5\%) a intervenção com medicina convencional mais a intervenção com medicina chinesa.

Das limitações metodológicas dos artigos selecionados, em nove artigos (53\%) o possível viés dado ao não cegamento foi explicitado como uma das limitações do estudo, uma vez que seria impossível cegar os 
participantes e profissionais da saúde já que as intervenções (com a MTC e a medicina convencional) são tão diferentes. Isto é, não se pode identificar com precisão os benefícios específicos da acupuntura (dada a falta de um grupo placebo como controle, característica dos ensaios pragmáticos).

Ainda das limitações, pode-se citar a pequena amostra populacional para avaliar a efetividade das intervenções. Além disso, havia em um artigo o viés de que a amostra populacional já tinha experiência prévia dos participantes com acupuntura - aqueles que já possuem confiança e experiência prévia com acupuntura tendem a responder ao tratamento mais positivamente (Itoh et al., 2008) - especialmente por serem estudantes de MTC e conhecerem a sensação de Qi.

Em quatro (24\%) dos artigos, foi explicitada a necessidade de uma amostra populacional maior, sendo que, entre esses, um ressaltou viés populacional por gênero, uma vez que $84 \%$ dos participantes eram mulheres; em dois outros artigos, haveria a necessidade de uma diversidade maior entre os participantes da amostra (em um deles, a amostra continha apenas estudantes universitários e na outra, os participantes eram estudantes de uma escola de acupuntura). No entanto, é o design de escolha determinar a relação custo-benefício (Moreira et al., 2013; Huf \& Kritski, 2012).

Quanto à duração das intervenções, 10 artigos (59\%) realizaram intervenções, semanais ou não, por até 12 semanas ou 3 meses, sendo que três artigos $(17,7 \%)$ incluídos nessa categoria continuaram um acompanhamento após a última intervenção de mais 12, 20 e 24 semanas, respectivamente.

Três artigos estudados relatam menor uso de analgésicos (dois desses mencionam menor prescrição médica de analgésicos) e medicação para dor para os grupos escolhidos aleatoriamente que receberam intervenção com acupuntura em relação ao grupo controle (GP) - um deles calculou uma taxa 15\% menor do uso de medicação entre os pacientes alocados para acupuntura em comparação ao que recebeu os cuidados convencionais (GP).

Os resultados a favor do tratamento com acupuntura nos artigos analisados se mostram mais relevantes quando utilizada a análise ao longo do tempo. Isso se evidencia por meio do uso de modelos longitudinais exploratórios que demonstraram melhorias lineares significativas ao longo do tempo na ansiedade, depressão, interferência na dor e distúrbios do sono para um dos artigos analisados (David et al., 2016).

Tabela 1. Artigos analisados e seus resultados.

\begin{tabular}{|c|c|c|c|c|c|c|}
\hline Ano & Artigo & $\mathbf{t}$ & $\mathbf{N}$ & Limitação & Intervenção & Principais resultados \\
\hline 2004 & $\begin{array}{c}\text { Acupuncture for chronic } \\
\text { headache in primary } \\
\text { care: }[\ldots]\end{array}$ & A & 401 & $\begin{array}{l}\text { Os pacientes do } \\
\text { estudo não foram } \\
\text { cegados. }\end{array}$ & $\begin{array}{l}\text { Pacientes } \\
\text { randomizados para } \\
\text { (a) acupuntura (por } \\
3 \text { meses) ou (b) } \\
\text { intervenção controle } \\
\text { de cuidado habitual. }\end{array}$ & $\begin{array}{c}\text { Pacientes randomizados para } \\
\text { acupuntura usaram } 15 \% \text { menos } \\
\text { medicação, fizeram } 25 \% \text { menos } \\
\text { visitas a médicos de clínica geral } \\
\text { e tiraram } 15 \% \text { menos dias de } \\
\text { folga. }\end{array}$ \\
\hline 2007 & $\begin{array}{l}\text { The effect of TCM } \\
\text { acupuncture on hot } \\
\text { flushes among } \\
\text { menopausal women } \\
\text { study: [...] }\end{array}$ & A & 286 & Estudo piloto. & $\begin{array}{l}\text { Grupo } 1: 10 \text { sessões } \\
\text { de tratamento com } \\
\text { acupuntura e } \\
\text { autocuidado; grupo } \\
\text { controle: } \\
\text { autocuidado. }\end{array}$ & - \\
\hline 2008 & $\begin{array}{l}\text { Effects of tender point } \\
\text { acupuncture on delayed } \\
\text { onset muscle soreness } \\
\text { (DOMS) - a pragmatic } \\
\text { trial. }\end{array}$ & A & 30 & $\begin{array}{c}\text { Pacientes } \\
\text { conhecedores de } \\
\text { acupuntura e da } \\
\text { sensação de deqi; } \\
\text { tinham confiança } \\
\text { na acupuntura }\end{array}$ & $\begin{array}{l}\text { Indivíduos foram } \\
\text { aleatoriamente } \\
\text { designados para os } \\
\text { grupos. }\end{array}$ & $\begin{array}{l}\text { Os resultados mostram que a } \\
\text { acupuntura no ponto sensível } \\
\text { tem efeitos analgésicos (ativam } \\
\text { receptores sensitivos do tipo } \\
\text { polimodal), eficaz nas síndromes } \\
\text { de dor miofascial. }\end{array}$ \\
\hline 2009 & $\begin{array}{l}\text { Exploring integrative } \\
\text { medicine for back and } \\
\text { neck pain - a pragmatic } \\
\text { randomised clinical pilot } \\
\text { trial. }\end{array}$ & Q\&A & 80 & $\begin{array}{l}\text { Incompatibilidade } \\
\text { entre } \\
\text { documentação e } \\
\text { sistemas } \\
\text { eletrônicos de } \\
\text { registro de } \\
\text { pacientes. }\end{array}$ & $\begin{array}{l}\text { Pacientes } \\
\text { randomizados para } \\
\text { atendimento: (a) } \\
\text { protocolo } \\
\text { integrativo ou (b) } \\
\text { convencional. }\end{array}$ & $\begin{array}{l}\text { Vitalidade era a favor do IM; } \\
\text { incapacidade auto-avaliada e } \\
\text { estresse: pequenas diferenças } \\
\text { clínicas entre os grupos que } \\
\text { apoiavam o IM; IM contribuiu } \\
\text { para menor uso de analgésico. }\end{array}$ \\
\hline 2012 & $\begin{array}{l}\text { Acupuncture for } \\
\text { depression: exploring } \\
\text { model validity and the } \\
\text { related issue of credibility } \\
\text { in the context of designing } \\
\text { a pragmatic trial. }\end{array}$ & A & 40 & $\begin{array}{l}\text { Estudo piloto, não } \\
\text { há possibilidade } \\
\text { de cegueira dos } \\
\text { pacientes. Eles } \\
\text { escolheram a } \\
\text { intervenção a } \\
\text { receber. }\end{array}$ & $\begin{array}{l}\text { Pacientes alocados } \\
\text { por preferência: } \\
62,5 \% \text { à acupuntura, } \\
\text { classificando-a } \\
\text { preferível de início a } \\
\text { aconselhamento ou } \\
\text { cuidados habituais. }\end{array}$ & $\begin{array}{l}\text { A estrutura do Conselho de } \\
\text { Pesquisa Médica (MRC), com sua } \\
\text { abordagem de método misto em } \\
\text { fases, ajudou a desenvolver } \\
\text { pesquisas com melhor validade } \\
\text { de modelo do que os ensaios } \\
\text { realizados até hoje neste campo. }\end{array}$ \\
\hline
\end{tabular}




\begin{tabular}{|c|c|c|c|c|c|c|}
\hline 2013 & $\begin{array}{l}\text { Moxibustion for treating } \\
\text { knee osteoarthritis: study } \\
\text { protocol of a multicentre } \\
\text { randomised controlled } \\
\text { trial }\end{array}$ & $\mathrm{M}$ & 212 & $\begin{array}{l}\text { Viés em relação à } \\
\text { cegueira; } \\
\text { tratamento } \\
\text { convencional é } \\
\text { grupo controle e } \\
\text { não procedimento } \\
\text { falso (placebo). }\end{array}$ & $\begin{array}{l}\text { Participantes } \\
\text { randomizados para } \\
\text { moxabustão }(12 \\
\text { sessões, } 3 \text { por } \\
\text { semana) ou apenas } \\
\text { cuidados usuais. }\end{array}$ & $\begin{array}{c}\text { Contribui para o } \\
\text { desenvolvimento de evidências } \\
\text { para a eficiência e segurança da } \\
\text { moxabustão para tratamento da } \\
\text { osteoartrite do joelho. }\end{array}$ \\
\hline 2013 & $\begin{array}{l}\text { Acupuncture for } \\
\text { Treatment of } \\
\text { Uncontrolled Pain in } \\
\text { Cancer Patients: A } \\
\text { Pragmatic Pilot Study }\end{array}$ & $\mathrm{A}$ & 48 & $\begin{array}{l}\text { Não houve } \\
\text { controle por } \\
\text { placebo; não } \\
\text { houve } \\
\text { acompanhamento } \\
\text { a longo prazo }\end{array}$ & $\begin{array}{l}\text { Os participantes com } \\
\text { dor } \geq 4(0-10) \\
\text { receberam no máximo } \\
10 \text { tratamentos de } \\
\text { acupuntura } \\
\text { individualizada. }\end{array}$ & $\begin{array}{l}\text { Os analgésicos prescritos } \\
\text { diminuíram; } 87 \% \text { tiveram } \\
\text { expectativas atendidas; } 90 \% \\
\text { participariam novamente; } 95 \% \\
\text { recomendariam acupuntura; e } \\
\text { 90\% a consideraram "útil" ou } \\
\text { "muito útil". }\end{array}$ \\
\hline 2014 & $\begin{array}{l}\text { A six-month effects of } \\
\text { integrative treatment, } \\
\text { therapeutic acupuncture } \\
\text { and conventional } \\
\text { treatment in alleviating } \\
\text { psychological distress in } \\
\text { primary care patients [...] }\end{array}$ & $\mathrm{A}$ & 154 & $\begin{array}{l}\text { A maioria (84\%) } \\
\text { dos pacientes era } \\
\text { do sexo feminino. }\end{array}$ & $\begin{array}{l}\text { Randomizados (6 - } 8 \\
\text { semanas) para } \\
\text { tratamento } \\
\text { integrativo (TI), } \\
\text { acupuntura (AT) e } \\
\text { tratamento } \\
\text { convencional (CT). } \\
\text { As avaliações foram } \\
\text { feitas na linha de } \\
\text { base após 8 e } 24 \\
\text { semanas. }\end{array}$ & $\begin{array}{c}\text { Às } 24 \text { semanas, TI e AT } \\
\text { apresentaram valores } \\
\text { significativamente melhores que } \\
\text { CT em todas as variáveis. }\end{array}$ \\
\hline 2014 & $\begin{array}{l}\text { Factors influencing } \\
\text { further acupuncture } \\
\text { usage and a more positive } \\
\text { outcome in patients with } \\
\text { osteoarthritis of the knee } \\
\text { and the hip: [...] }\end{array}$ & A & 202 & $\begin{array}{l}\text { Não houve } \\
\text { intervenção } \\
\text { fraudulenta } \\
\text { (placebo); grupo } \\
\text { controle também } \\
\text { recebeu } \\
\text { acupuntura. }\end{array}$ & $\begin{array}{c}\text { Pacientes } \\
\text { randomizados para } \\
\text { acupuntura imediata } \\
\text { nos primeiros } 3 \\
\text { meses ou } \\
\text { acupuntura nos } 3 \\
\text { meses subsequentes }\end{array}$ & $\begin{array}{c}\text { Após } 36 \text { meses, o Índice } \\
\text { WOMAC não diferiu } \\
\text { significativamente entre os } \\
\text { grupos (acupuntura imediata vs. } \\
\text { acupuntura tardia. }\end{array}$ \\
\hline 2015 & $\begin{array}{l}\text { Acupuncture for the } \\
\text { treatment of phantom } \\
\text { limb pain in lower limb } \\
\text { amputees: }[. . .]\end{array}$ & A & 20 & $\begin{array}{l}\text { Amostra pequena: } \\
\text { ênfase na } \\
\text { viabilidade, não } \\
\text { na significância } \\
\text { estatística dos } \\
\text { resultados }\end{array}$ & $\begin{array}{c}\text { Participantes } \\
\text { aleatorizados para } \\
\text { cuidados usuais ou } \\
\text { os cuidados usuais } \\
\text { mais a acupuntura } \\
\text { (8 tratamentos por } 4 \\
\text { semanas). }\end{array}$ & $\begin{array}{l}\text { Quantitativamente, a } \\
\text { acupuntura demonstrou } \\
\text { mudança clinicamente } \\
\text { significativa na intensidade } \\
\text { média da dor e da pior } \\
\text { intensidade de dor. }\end{array}$ \\
\hline 2016 & $\begin{array}{c}\text { Acupuncture-Related } \\
\text { Quality of Life Changes } \\
\text { Using PROMIS Computer } \\
\text { Adaptive Tests in a } \\
\text { Pragmatic Trial with } \\
\text { Oncology and General IM } \\
\text { Patients: [...] }\end{array}$ & $\mathrm{A}$ & 81 & $\begin{array}{l}\text { Amostra pequena; } \\
\text { atrito entre os } \\
\text { dados da } \\
\text { avaliação ao } \\
\text { longo do tempo. }\end{array}$ & $\begin{array}{l}\text { Interessados } \\
\text { receberam } \\
\text { acupuntura. Pontos } \\
\text { de acupuntura } \\
\text { variaram } \\
\text { dependendo dos } \\
\text { sintomas do } \\
\text { paciente. }\end{array}$ & $\begin{array}{l}\text { Entre T1 e T2, todos os } \\
\text { participantes relataram } \\
\text { melhorias significativas na } \\
\text { ansiedade, depressão e fadiga. } \\
\text { Modelos exploratórios } \\
\text { demonstraram melhorias ao } \\
\text { longo do tempo na ansiedade, } \\
\text { depressão, dor e sono. }\end{array}$ \\
\hline 2016 & $\begin{array}{l}\text { Semi-individualised } \\
\text { Chinese medicine } \\
\text { treatment as an adjuvant } \\
\text { management for diabetic } \\
\text { nephropathy: }[. . .]\end{array}$ & $\mathrm{F}$ & 148 & $\begin{array}{l}\text { Estudo piloto: } \\
\text { tamanho da } \\
\text { amostra é não é } \\
\text { alimentado para } \\
\text { alcançar } \\
\text { significância } \\
\text { estatística. }\end{array}$ & $\begin{array}{l}\text { Pacientes } \\
\text { randomizados } 1: 1 \\
\text { para tratamento de } \\
\text { MTC adicional (48 } \\
\text { semanas) ou } \\
\text { atendimento } \\
\text { padrão. }\end{array}$ & - \\
\hline 2017 & $\begin{array}{c}\text { Study protocol of a } \\
\text { pragmatic, randomised } \\
\text { controlled pilot trial: } \\
\text { clinical effectiveness on } \\
\text { smoking cessation of TCM } \\
{[\ldots]}\end{array}$ & $\mathrm{A}$ & 40 & $\begin{array}{l}\text { Viés devido ao } \\
\text { desenho não cego } \\
\text { do estudo; Estudo } \\
\text { piloto com um } \\
\text { pequeno tamanho } \\
\text { de amostra. }\end{array}$ & $\begin{array}{l}\text { Participantes } \\
\text { randomizados para } \\
\text { acupuntura, além da } \\
\text { reposição de } \\
\text { nicotina, ou para só } \\
\text { reposição, } 4 \\
\text { semanas de } \\
\text { tratamento, } 20 \text { de } \\
\text { acompanhamento. }\end{array}$ & - \\
\hline 2017 & $\begin{array}{l}\text { Standardised versus } \\
\text { individualised multiherb } \\
\text { Chinese herbal medicine } \\
\text { for oligomenorrhoea and } \\
\text { amenorrhoea in } \\
\text { polycystic ovary }\end{array}$ & $\mathrm{F}$ & 40 & $\begin{array}{l}\text { Na avaliação do } \\
\text { hirsutismo não } \\
\text { foram levantadas } \\
\text { a taxa de } \\
\text { crescimento } \\
\text { capilar nem a área }\end{array}$ & $\begin{array}{l}\text { Mulheres } \\
\text { randomizadas para } \\
\text { intervenção de } 6 \\
\text { meses de } \\
\text { fitoterápico chinês } \\
\text { padronizado ou }\end{array}$ & $\begin{array}{l}\text { Houve melhoria nas taxas } \\
\text { menstruais para ambos os } \\
\text { grupos (padronizados e } \\
\text { individualizados). Melhorias nos } \\
\text { escores de hirsutismo não foram } \\
\text { estatisticamente significantes. }\end{array}$ \\
\hline
\end{tabular}




\begin{tabular}{|c|c|c|c|c|c|c|}
\hline & syndrome:[...] & & & $\begin{array}{l}\text { de distribuição } \\
\text { capilar. }\end{array}$ & $\begin{array}{l}\text { individualizado } \\
\text { (posologia 16g } \\
\text { diários por via oral - } \\
\text { chá). }\end{array}$ & $\begin{array}{l}\text { Os dados de função hepática e } \\
\text { renal e eventos adversos foram } \\
\text { amplamente normais. }\end{array}$ \\
\hline 2017 & $\begin{array}{l}\text { Moxibustion for pain } \\
\text { relief in patients with } \\
\text { primary dysmenorrhea: } A \\
\text { randomized controlled } \\
\text { trial. }\end{array}$ & M & 133 & $\begin{array}{c}\text { Medidas } \\
\text { subjetivas para } \\
\text { medir níveis de } \\
\text { dor; não houve } \\
\text { cegamento; } \\
\text { participantes } \\
\text { eram em maioria } \\
\text { estudantes } \\
\text { universitários. }\end{array}$ & $\begin{array}{l}\text { Participantes } \\
\text { randomizados em } \\
\text { média para (a) } \\
\text { moxabustão ou (b) } \\
\text { medicamento } \\
\text { convencional } \\
\text { (ibuprofeno) - } 3 \\
\text { meses. }\end{array}$ & $\begin{array}{c}\text { A intensidade da dor foi } \\
\text { significativamente reduzida em } \\
\text { ambos. Três meses após o } \\
\text { tratamento, a eficiência da } \\
\text { moxabustão foi maior do que a } \\
\text { da droga. }\end{array}$ \\
\hline 2017 & $\begin{array}{c}\text { Acupuncture in Patients } \\
\text { with Allergic Asthma: A } \\
\text { Randomized Pragmatic } \\
\text { Trial. }\end{array}$ & A & $\begin{array}{l}14 \\
45\end{array}$ & $\begin{array}{l}\text { Não houve } \\
\text { cegamento para } \\
\text { pacientes ou } \\
\text { médicos; viés de } \\
\text { aceitação social; } \\
\text { sem placebo }\end{array}$ & $\begin{array}{l}\text { Pacientes } \\
\text { randomizados e não } \\
\text { randomizados para } \\
\text { (a) acupuntura (até } \\
15 \text { sessões em } 3 \\
\text { meses) ou (b) } \\
\text { cuidados de rotina. }\end{array}$ & $\begin{array}{l}\text { Na parte randomizada, a } \\
\text { acupuntura foi associada a uma } \\
\text { melhora na escala do } \\
\text { componente físico e a escala de } \\
\text { componentes mentais em } \\
\text { comparação ao grupo controle }\end{array}$ \\
\hline 2019 & $\begin{array}{l}\text { Traditional Chinese } \\
\text { medicine (TCM) } \\
\text { collaborative care for } \\
\text { patients with axial } \\
\text { spondyloarthritis } \\
\text { (AcuSpA): protocol for a } \\
\text { PRCT. }\end{array}$ & A & 160 & Estudo piloto. & $\begin{array}{c}\text { Pacientes } \\
\text { randomizados 1:1 } \\
\text { para os cuidados } \\
\text { reumatológicos } \\
\text { usuais ou } \\
\text { intervenção com } \\
\text { MTC (10 sessões de } \\
30 \text { minutos) }\end{array}$ & $\begin{array}{l}\text { Este estudo pode fornecer } \\
\text { evidências sobre a eficácia } \\
\text { clínica, segurança e custo- } \\
\text { efetividade de um TCMCMC } \\
\text { para pacientes com AxSpA. }\end{array}$ \\
\hline
\end{tabular}

$\mathrm{t}=$ Terapia testada $\mathrm{N}$ = tamanho da amostra $\mathrm{A}=$ acupuntura $\mathrm{F}$ = fitoterapia; $\mathrm{M}=$ moxabustão.

\section{Os princípios da Medicina Chinesa, diagnóstico e tipos de tratamento}

A Medicina Tradicional Chinesa considera que existem diversos meridianos (canais por onde circula a energia vital $Q i$ ) espalhados pelo corpo. Dessa maneira, a MTC se caracteriza por uma racionalidade médica vitalista (Souza \& Luz, 2011; Ferreira \& Luz, 2007), que possui o conceito de vitalidade e energia vital. Desses canais, existem 12 principais que percorrem os membros inferiores e superiores e estão conectados internamente com nossos órgãos. É uma medicina que baseia seu tratamento e diagnóstico no princípio yin e yang - os quais se manifestam nos 5 movimentos essenciais da natureza, representados por: ar, água, metal, madeira e fogo (Lu et al., 2004; Maciocia, 2006). Esses movimentos se apresentam tanto no biotipo da pessoa quanto no funcionamento de cada órgão. Cada pessoa e órgão saudáveis possuem os princípios yin e yang em equilíbrio - o qual pode ser alcançado através de técnicas da MTC e restaurar a saúde. Também se considera que, além do Qi, circulam pelo nosso corpo 5 substâncias vitais, que nos nutrem, nos lubrificam, transportam nutrientes e energia às células e nos mantém vivos (são elas Xue, Jin Ye, Jing, Qi, Shen) (Ross, 1994; Wang et al., 2016; Chan, 1995; Maciocia, 2006).

Isto é, existem várias maneiras e óticas diferentes quando olhamos para o indivíduo a ser tratado pela MTC, não podendo separar corpo de mente. É uma medicina complexa, que considera o indivíduo como um todo: um organismo singular e integral, cujas partes não podem ser separadas em tratamento.

Dentro desse contexto, a doença se define na MTC como a destruição do equilíbrio entre o interior do ser humano (órgãos), dentre vários tipos de fatores / elementos, e entre a pessoa e seu ambiente. Isto dá origem a uma condição de estrutura e funcionamento anormais, bem como uma incapacidade de se recuperar rapidamente por conta própria (Lu et al., 2004; Wang et al., 2016).

A MTC possui diferentes sistemas de diagnóstico e tratamento e deve-se entender que as doenças dentro desses sistemas aparecem devido a fatores adoecedores diferentes (Zhufan, 2008; Maciocia \& Ming, 1996; Ross, 1994; Chan, 1995). Por exemplo, há fatores adoecedores ligados a Zàng-fu (órgãos e vísceras), Jing-Luò (meridianos) e estruturais.

O diagnóstico é feito partindo do princípio de que a doença pode ter sido contraída por um dos (a) fatores externos (Zhufan, 2008; Maciocia \& Ming, 1996; Ross, 1994; Silva, 2007): vento, secura, umidade, calor, frio, fogo - pensava-se o corpo humano como um microcosmo da natureza, que poderia ser atacado por 'excessos' de determinado tipo, que invadem o sistema Zang-fu (12 órgãos e vísceras principais no corpo humano, cada um cumprindo funções em níveis: fisiológico, vital e mental-emocional). 
A doença poderia ser contraída também por (b) fatores internos (Silva, 2007), em que determinado tipo de emoções seria gerado pela atividade da força vital nos órgãos do corpo humano (medo, pânico, raiva, preocupação, euforia, tristeza - cada uma delas afetaria um órgão). As emoções seriam capazes de gerar desequilíbrios fisiológicos, por atividade excessiva ou incapacidade organizativa do sistema.

Por fim, por (c) mistos (Silva, 2007): hábitos singularizados não adequados à constituição do indivíduo, relacionados à alimentação (preparo e tipo de alimentos), ao sono (relacionado ao excesso de trabalho e exaustão física) e à sexualidade (indulgência sexual ou atividade excessiva, desgastante).

A gênese das enfermidades, em termos gerais, era pensada via interação da capacidade adaptativa do ser individual com os ciclos da natureza, fundamentada nas concepções cosmológicas que têm no homem e no cosmo um conjunto integrado pela força vital Qì (Ross, 1994; Silva, 2007; Cordeiro \& Cordeiro, 1992; Maciocia, 2006). Ao serem analisadas as queixas, o organismo de indivíduo como um todo, sua interação com o ambiente e com os outros e seu estilo de vida, o médico chinês ou acupunturista preverá uma "síndrome" (nome específico do estado patológico do organismo) dentro da MTC que levará a um princípio específico de tratamento.

O tratamento segue tal princípio e se utiliza das técnicas de acupuntura, acupressão, moxabustão; terapia medicamentosa (uso de plantas, animais e minerais); prática corporal (qi kong); massagens Shiatsu e Tui Ná; dietética.

\section{A metodologia pragmática atende aos princípios da MTC?}

Como explicado brevemente, a MTC é uma medicina que considera o ser humano como um ser em constante mudança, influenciado pela natureza (através dos 5 movimentos, fatores patógenos, alimentação, etc.); como um ser que possui sua singularidade em todos os níveis de sua existência e cujo conjunto mentecorpo-espírito (shen) não pode ser fragmentado - visão integral (Ferreira \& Luz, 2007). É uma medicina que considera o conceito de vitalidade como um dos pilares para identificar a saúde do organismo vivo e possui um complexo sistema de diagnóstico.

O princípio básico utilizado pelo profissional da saúde que atua com Medicina Chinesa é o de reduzir o desequilíbrio e restaurar a vitalidade daquele organismo. Nesse sentido, quando analisadas as queixas e estilo de vida do paciente, o profissional dirá uma síndrome cujo princípio de tratamento (Cordeiro \& Cordeiro, 1992; Maciocia, 2006) agirá de maneira alopática a ela. Por exemplo, se considera que o organismo analisado tem "excesso de calor no fígado" ou "excesso de yang do fígado", o princípio de tratamento será reduzir calor no fígado ou aumentar o yin do fígado, dependendo da condição do indivíduo.

Como se considera o indivíduo como um todo, cujas partes não podem ser separadas e cujos Zàng Fu (órgãos e vísceras) possuem não só ação fisiológica (Maciocia, 2006), mas relação com aspectos mentaisemocionais e com as substâncias vitais, entende-se o quão difícil seria aplicar uma metodologia como a RCT (padrão ouro) às medicinas alternativas e complementares (Andrade \& Portella, 2018) devido à impossibilidade de realizar um estudo duplo-cego em algumas intervenções e à abordagem individualizada e complexa dessas práticas.

O que a PRCT traz de benefícios metodológicos aos ensaios clínicos sobre as terapêuticas da MTC, em termos de melhorar a análise de resultados e compreender sua eficiência, acontece em função de que não excluir a complexidade da terapêutica e a visão singular do profissional da saúde em relação aos pacientes tratados na pesquisa, já que mimetiza a prática clínica como ela é. Os ensaios que utilizam PRCT superam os obstáculos trazidos pela RCT, de modo que a análise de subgrupos de diagnóstico em terapias de CAM, como as síndromes na MTC, podem ser usadas nesses estudos, melhorando a relação analítica entre dados qualitativos e componentes quantitativos (Andrade \& Portella, 2018).

Percebemos existir a necessidade pesquisas com métodos em ensaios clínicos que avaliem a qualidade dos tratamentos da MTC, como os ensaios randomizados explanatórios, controlados por placebo, quando possível. Tais ensaios certamente produzem um nível mais alto de evidência, mas, dado o alto custo e complexidade, devem ser implementados quando evidências pragmáticas favoráveis forem acumuladas. Esses estudos podem estabelecer variabilidade nos resultados primários para permitir o cálculo do tamanho da amostra para um estudo em grande escala e explorar os critérios de viabilidade e design (Reynolds et al., 2008).

O que se observa a partir dos resultados observados é o alto nível de resultado em favor da MTC com o uso da metodologia pragmática (em comparação com o tratamento usual biomédico). Em resumo, percebemos sua relevância em termos de complementaridade à medicina convencional, satisfação dos pacientes, redução dos sintomas e redução do uso de medicamentos alopáticos (prescritos ou não). Isso é importante para provar 
sua eficiência e ganhar notoriedade científica e clínica e justificar a elaboração de ensaios clínicos maiores (de amostra grande). Apesar de não se conhecer seus efeitos específicos a partir de tal metodologia, mostrase como a melhor metodologia em termos de custo-benefício (Moreira et al., 2013).

\section{A metodologia pragmática é adequada para estudar a MTC?}

Os ensaios pragmáticos procuram descrever a efetividade da intervenção, ou seja, seu resultado em condições que mimetizam a prática clínica e com dificuldades de decisão (Huf \& Kritski, 2012). Os termos eficácia e efetividade são geralmente usados para caracterizar os objetivos dos tipos de abordagem (Coutinho \& Bloch, 2003), eficácia de uma intervenção é percebida por ensaios exploratórios, por exemplo, e se refere aos mecanismos de ação de tal prática terapêutica.

A abordagem pragmática é pertinente quando não é possível conduzir uma investigação clínica em um ambiente puramente experimental, o que é o caso das PIC, pois tratam-se de terapias embasadas em conhecimentos tradicionais e não passíveis de redução. Para as racionalidades médicas vitalistas, a pesquisa clínica e a prática clínica são indissociáveis e têm como eixo central, a saúde do paciente e a maior efetividade das ações (Coutinho \& Bloch, 2003; Ferreira \& Luz, 2007). Dessa forma, a PRCT parece ser uma um método muito adequado para estudar cientificamente a eficiência da MTC e das PIC, de forma que respeita a abordagem complexa das medicinas tradicionais, como a chinesa, e acomodam aspectos individuais dos pacientes, possibilitando uma amostra heterogênea.

\section{Considerações finais}

Quando se busca a efetividade de um tratamento, maior custo-benefício ou não se deseja realizar o estudo em um ambiente puramente experimental, a pRCT é adequada. Em MTC, a pRCT se faz adequada, já que a MTC utiliza de uma visão multidimensional (não se busca a especificidade no tratamento) e singular do sujeito e tal metodologia oferece o benefício de não excluir a complexidade da terapêutica e a visão singular do profissional da saúde em relação aos pacientes tratados na pesquisa.

\section{Referências}

Andrade, F. A., \& Portella, C. F. S. 2018. Research methods in complementary and alternative medicine: an integrative review. Journal of Integrative Medicine, 16(1), 6-13.

BRASIL. 2006. Política Nacional de Práticas Integrativas e Complementares no SUS (PNPIC-SUS). Brasília, DF: Ministério da Saúde.

Contatore, O. A., Barros, N. F., Durval, M. R., Barrio, P. C. C. C., Coutinho, B. D., Santos, J. A., Nascimento, J. L., Oliveira, S. L., \& Peres, S. M. P. 2015. Uso, cuidado e política das práticas integrativas e complementares na Atenção Primária à Saúde. Ciência \& Saúde Coletiva, 20(10), 3263-3273.

Cordeiro, A. T., \& Cordeiro, R. C. 1992. Acupuntura elementos básicos. São Paulo: Ensaio, 121p.

Coutinho, E. S. F., Huf, G., \& Bloch, K. V. 2003. Ensaios clínicos pragmáticos: uma opção na construção de evidências em saúde. Cadernos de Saúde Pública, 19(4), 1189-1193.

Chan, K. 1995. Progress in traditional Chinese medicine. Trends in Pharmacological Sciences, 16(6), 182-187.

David, V., Beaumont, J. L., Rupa, M., Grimone, A., Burns, V., Murry, W., Gutierrez, S., Schuette, S., Brady, C., \& Ring, M. 2016. Acupuncture-Related Quality of Life Changes Using PROMIS Computer Adaptive Tests in a Pragmatic Trial with Oncology and General Integrative Medicine Patients: The Role of Baseline Acupuncture Expectations. The Journal of Alternative and Complementary Medicine, 22(10), 778-787.

Ercole, F. F., Melo, L. S., Alcoforado, CLGC. 2014. Integrative review versus systematic review. Revista Mineira de Enfermagem, 18(1), 1-260.

Ferreira, C. S., \& Luz, M. T. 2007. Shen: categoria estruturante da racionalidade médica chinesa. História, Ciências, Saúde-Manguinhos, 14(3), 863-875.

Huf, G., \& Kritski, A. 2012. Avaliação da utilidade clínica de novos testes diagnósticos em tuberculose: o papel dos ensaios clínicos pragmáticos. Jornal Brasileiro de Pneumologia, 38(2), 237-245.

Itoh, K., Ochi, H., \& Kitakoji, H. 2008. Effects of tender point acupuncture on delayed onset muscle soreness (DOMS) - a pragmatic trial. Chinese Medicine, 3(14). 
Lu, A.-P., Jia. H.-W., Xiao, C., \& Lu, Q.-P. 2004. Theory of traditional Chinese medicine and therapeutic method of diseases. World Jorunal of Gastroenterolohy, 10(13), 1854-1856.

MacPherson, H. 2004. Pragmatic clinical trials. Complementary Therapies in Medicine, 12(2-3), 136-140.

Maciocia, G., \& Ming, S. X. 1996. Os fundamentos da medicina chinesa: um texto abrangente para acupunturistas e fitoterapeutas. São Paulo: Roca, 658p.

Maciocia, G. 2006. Diagnóstico na Medicina Chinesa: um guia geral, São Paulo: Roca.

Moreira, A. S. R, Huf, G., Vieira, M. A., Fonseca, L., Ricks, M., \& Kritski, A. L. 2013. Performance comparison between the mycobacteria growth indicator tube system and Löwenstein-Jensen medium in the routine detection of Mycobacterium tuberculosis at public health care facilities in Rio de Janeiro, Brazil: preliminary results of a pragmatic clinical trial. Jornal Brasileiro de Pneumologia, 39(3), 365-367.

Resch, K. L. 1998. Pragmatic Randomised Controlled Trials for Complex Therapies. Forsch Komplementarmed, 5(Suppl 1), 136-139.

Reynolds, J. A., Bland, J. M., \& MacPherson, H. 2008. Acupuncture for Irritable Bowel Syndrome - An Exploratory Randomised Controlled Trial. Acupuncture in Medicine, 26(1), 8-16.

Ross, J. 1994. Zang Fu: Sistemas de órgãos e vísceras da medicina. 2.ed. São Paulo: Roca, 11p.

Rocha, S. P., \& Gallian, D. M. C. 2013. Uma nova abordagem dos estudos da medicina tradicional chinesa no Ocidente. Physis: Revista de Saúde Coletiva, 23(3), 995-1001.

Roland, M., \& Torgerson, D. J. 1998. Understanding controlled trials: What are pragmatic trials? The BMJ, 316.

Silva, D. F. 2007. Psicologia e acupuntura: aspectos históricos, políticos e teóricos. Psicologia: Ciência e Profissão, 27(3), 418-429.

Souza, E. F. A. A., \& Luz, M. T. 2011. Análise crítica das diretrizes de pesquisa em medicina chinesa. História, Ciências, Saúde-Manguinhos, 18(1), 155-174.

Tabosa, A. 2012. Pesquisas em medicina tradicional chinesa. Revista Brasileira de Medicina de Família e Comunidade, 7(1), 9.

Zhufan, X. 2008. Prática de Medicina Chinesa. 1.ed. São Paulo: Î́cone.

Wang, L., Qiao, K., Zhang, H., \& Zhang, K. 2016. Chinese medicine and environmental disease. Environmental Disease, 1(3), 95-98.

Ware, J. H., \& Hamel, M. B. 2011. Pragmatic trials--guides to better patient care? The New England Journal of Medicine, 364(18), 1685-1687.

\section{Minicurrículo}

Bruna Verzili Gallo. Mestranda em Neurociência e Cognição na Universidade Federal do ABC. Possui graduação em Naturologia pela Universidade Anhembi Morumbi (2020); Professora de Hatha Yoga e meditação (2016 - atual); Atua com Práticas Integrativas e Complementares. Tem experiência em Saúde da Mulher dentro do Programa de Saúde da Criança e do Adolescente, atuando como Naturóloga voluntária. Participa do Geanm - Grupo de Estudos de Aspectos Neuropsiquiátricos e Motricidade da UFABC e pesquisa os efeitos do Hatha Yoga sobre aspectos neuropsiquiátricos de idosos.

Ana Claudia de Leite-Mor. Bacharel em Naturologia Aplicada pela Universidade do Sul de Santa Catarina (UNISUL) em 2010. Mestre em Saúde Coletiva pela Universidade Estadual de Campinas (UNICAMP) em 2014. Especialista em Práticas Corporais da Medicina Tradicional Chinesa - Foco em Tai Chi Chuan da Familia Yang pela EACH-USP e SBTCC. No mestrado dedicou-se ao estudo da democratização da saúde pela perspectiva da antropologia de Bruno Latour. Desenvolveu estudos sobre a Política Nacional de Práticas Integrativas e Complementares (PNPIC), sobre a mudança de paradigma em saúde e sobre a fundamentação teóricoepistemológica da Naturologia. Atualmente é docente do curso de Naturologia da Universidade Anhembi Morumbi (UAM). Desenvolve pesquisas nas áreas de epistemologia, diálogo ocidente-oriente, práticas corporais integrativas, medicinas tradicionais brasileiras e nas filosofias antigas ocidentais e orientais. 
Como citar: Gallo, B.V., \& Leite-Mor, A.C. 2021. A viabilidade da metodologia Pragmatic Randomized Clinical Trials (PRCT) à pesquisa de Medicina Tradicional Chinesa. Pubsaúde, 5, a085. DOI: https://dx.doi.org/10.31533/pubsaude5.a085

Recebido: 8 out. 2020.

Revisado e aceito: 28 out. 2020.

Conflito de interesse: os autores declaram, em relação aos produtos e companhias descritos nesse artigo, não ter interesses associativos, comerciais, de propriedade ou financeiros que representem conflito de interesse.

Licenciamento: Este artigo é publicado na modalidade Acesso Aberto sob a licença Creative Commons Atribuição 4.0 (CC-BY 4.0). 